\title{
Measurement of Temperature Dependent Dielectric Constant of Coal Samples for Burden Surface Profiling at Blast Furnace
}

\author{
Chitresh Kund $u^{1, *}$, Prabal Patra ${ }^{2}$, Bipan Tudu ${ }^{3}$, Dibyayan Patra ${ }^{4}$ \\ ${ }^{1}$ TATA Steel, Instrumentation \& Control, Jamshedpur, Jharkhand, India. \\ ${ }^{2}$ TATA Steel, Instrumentation \& Control, Jamshedpur, Jharkhand, India. \\ ${ }^{3}$ Jadavpur University, Kolkata, West Bengal, India. \\ ${ }^{4}$ Vellore Institute of Technology, Vellore, Tamil Nadu, India. \\ * email:chitresh.kundu@tatasteel.com
}

DOI: https://doi.org/10.30609/jeti.v4i2.12584

\begin{abstract}
Blast furnaces (BFs) are the key receptacles of iron and steel smelting. Iron ore, coke and limestone are some of the raw materials used in iron making. The charging operation needs to be accomplished by accurately estimating the current depth of the burden surface. To achieve the goal of global class steel production, burden profile measurement and monitoring are vital. This measuring and monitoring help in augmenting the best usage of charge materials and energy consumptions. Radar-based measurement is best for determining the level and profile of the burden inside the furnace. However, for the optimal operation of the radar, it is essential to know the dielectric constant of the material. There are many approaches to determine the dielectric constant like capacitive methods, transmission line methods, cavity resonator methods, open cavity methods, etc. For this study, the cavity resonator method was used for measuring the permittivity of coal samples. The reflection capability of electromagnetic waves by coal depends on its dielectric properties which also depends on temperature. The results presented in this paper provide essential design input for radar-based measurements at the blast furnace, mainly for burden profiling at blast furnaces.

Keywords: Dielectric constant, Coal, Cavity resonator, blast furnace
\end{abstract}

\section{Introduction}

A blast furnace is a smelting pot of ore of the metal, coke, and limestone along with high temperature and pressure [1]. The hot air blown from the bottom of the furnace heats these charged materials while it ascends. In the blast furnace, measuring the burden profile is one of the most important parameters related to process stability [2]. Burden profile contains alternate layers of ferrous material as iron ore and carbon sources like coke and 
coal. As the carbon layer will act as a dielectric medium the dielectric constant needs to be determined.

Microwave absorption and reflectivity are dependent on the dielectric properties of the medium and are essential to estimate electromagnetic wave reflection for radar-based measurement. The dry organic mass in coal presents low dielectric loss, and is essentially transparent to microwaves at room temperature. Some components with higher dielectric loss in the coal matrix, such as moisture and some minerals including pyrite, may increase the dielectric constant of coal. As the microwave properties of coal can get significantly affected by the temperature. Particularly the burden surface temperature may reach up to 500 degrees centigrade. Hence, the paper aims to study the microwave properties of coal through the characterization of the dielectric constant in a broad temperature range up to 600-degree centigrade.

\section{Materials and methods}

The measurements for permittivity or dielectric constant have been introduced and applied many years ago [2]. These properties integrate a central role in choice of frequency for the material, type and geometry of radiator. It also empowers to understand the change in material's microwave absorption characteristics during the operating process. Further in the designing and development of radars the dielectric constant values play an essential role to understand the echo properties, which in turn provides insight for imaging capability and potential to acquire subsurface information. The interaction of coal with microwaves and heating of coal mass by microwave depend on coal's dielectric properties. Hence, these properties of coals need to be measured for determining the optimum frequency of absorption for the phased array radar system.

Dielectric properties of the material to be measured are as follows:

- Dielectric constant $\left(\varepsilon^{\prime}\right)$ : Material property which indicates how much energy is stored in material when microwave is applied externally.

- Loss constant ( $\left({ }^{\prime \prime}\right)$ : Material property which indicates how much energy is lost from material in form of heat when microwave is applied externally.

$$
\varepsilon_{\mathrm{r}}=\varepsilon^{\prime}-i \varepsilon^{\prime}
$$

Where, $\varepsilon_{\mathrm{r}}$ is known as complex relative permittivity of material. 
- Loss tangent $(\tan \delta$ ): Ratio of $\varepsilon$ " and $\varepsilon$, tells how much energy is converted to heat as compared to stored or how effective is microwave heating [ $\left.\tan \delta=\varepsilon^{\prime \prime} / \varepsilon^{\prime}\right]$.

- Quality factor (Q): In context of material only (ignoring external factors in microwave setup), Quality factor is the inverse of Loss tangent $[\mathrm{Q}=1 / \tan \delta]$.

At microwave frequencies, the principal components that influence the reflection of waves are the dielectric properties of the materials. Regularly, the diverse dielectric materials will prompt the various losses and reflections for microwave frequencies. The assessment of the dielectric properties from the multiple materials serves as an essential input requirement in microwave design.

The earlier concept of the permittivity measurement is based on DC electrical resistance of the material [3]. However, with further development in microwave engineering, newer methods like waveguide, cavity resonator, free space, etc. are used widely as discussed in the next section

\subsection{Parallel Plate Capacitor}

The parallel plate capacitor is used as the test cell for measuring the dielectric samples. The complex dielectric permittivity is gained by measuring the change of capacitance and of conductance due to the device with and without specimen, the fringing field affections are explained by mathematical corrections. Figure 1 shows the measurement by using capacitor method.

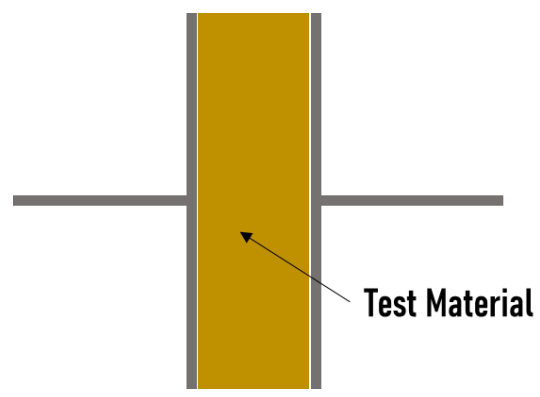

Figure 1 Parallel Plate Capacitor Measurement Method

\subsection{Transmission Line}

Transmission line method is suitable for the broadband frequencies, including the arrangement slab or annular geometry, as illustrated in Figure 2. The coaxial lines and waveguide are normally used to measure samples for the powder and liquid materials [4] and even for materials inside a partially filled waveguide [5]. 


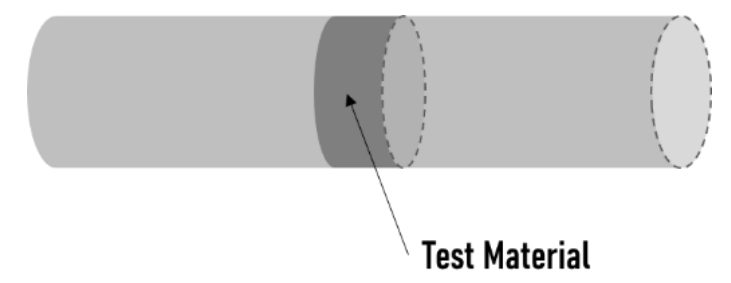

Figure 2 Transmission Line Methods

\subsection{Free Space Methods}

In free space method, the material to be tested are not required to specify the geometry, therefore this method can be used to measure the materials at high temperature[7]. It uses two antennas for transmitting and receiving, the material is placed between these two antennas, the sample usually fixed on a slab[8][9], see Figure 3. To determine the dielectric properties, the attenuation and phase shift are evaluated. On the other hand, the reflection measurements are also possible for free space method [10][11].

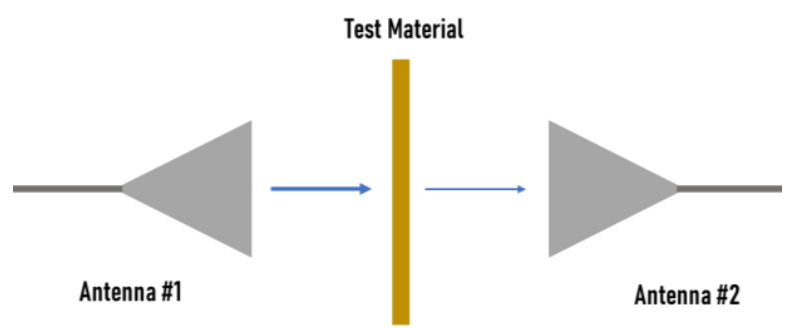

(a)

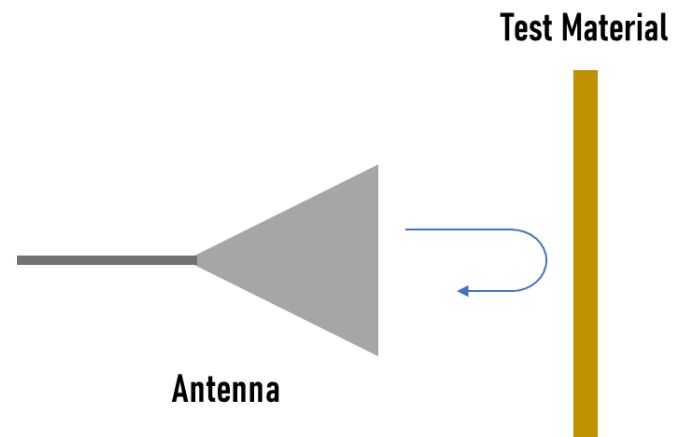

(b)

Figure 3 Free Space Method; (a) Transmission Measurement; (b) Reflection Measurement.

\subsection{Microwave Cavity Resonators}

The microwave cavity resonators as shown in Figure 4 are popularly used in measurement of dielectric constant for low permittivity materials. Using this method both the real and imaginary part of the permittivity can be obtained by measuring the resonance 
frequency of the empty and loaded cavity. This method has an upper edge for dielectric measurements and loss tangents over a wide range of frequencies. Hence, the method used for this paper.

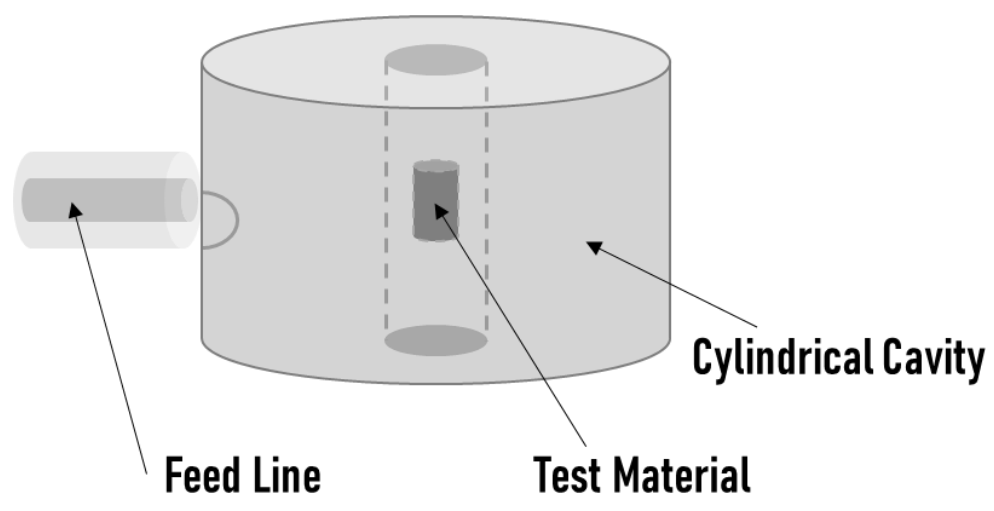

Figure 4 The Dielectric Material Measured by Cylindrical Cavity Resonator

Table 1 Comparison of these methods depending upon the permittivity of the material,

\begin{tabular}{ccc}
\hline & $\begin{array}{c}\text { Accuracy } \\
\text { (Low permittivity) }\end{array}$ & $\begin{array}{c}\text { Accuracy } \\
\text { (High permittivity) }\end{array}$ \\
\hline Capacitor Method & High & High \\
\hline Tx Line & Medium & Medium \\
\hline Cavity Method & High & Low \\
\hline Free Space & Medium & Medium \\
\hline
\end{tabular}

\subsection{Experimental Setup}

For our analysis, the cavity resonator-based method has been used. The general construction principle of the cavity resonator involves the circular waveguide design, which is short-circuited at opposite ends using metallic plates. The final design takes the shape of a cylindrical metal box. An illustration for the cylindrical cavity resonator is shown in figure 1. Inside the cavity, the electric and magnetic fields exist as per the excitation mode. The stored energies are the summation of the electric and magnetic fields within the cavity. The power dissipation takes place in the metallic wall and the dielectric material filling inside the cavity resonator. In our case, the metal wall is made up of copper, and the air is the dielectric inside. 
The excitation of the cavity is usually done using a coupling device. The power is fed from the measuring equipment, a vectored network analyzer, to the cavity through the coupling probe [12][13]. The type and loading effect of the coupling devices is different for the TE and TM modes.

\subsection{Design of cylindrical cavity}

The design started with the simulation of a cylindrical cavity resonator operating at TM010 using high frequency structure simulator (HFSS) software. 99.9\% high conductivity copper material has been used for fabrication. The inner surface has been coated with silver paint to decrease skin depth.

The cavity resonator thus designed resonates at $790 \mathrm{MHz}$. Figure 5 shows the copper cavity resonator dimensions used in the HFSS simulations. The radius of the cavity resonator is $150 \mathrm{~mm}$; the, height is $55 \mathrm{~mm}$, and the, deeding coupling loop is designed on the middle of the metal wall with a radius of $5 \mathrm{~mm}$. Corresponding to the TM010 mode, the electric and magnetic fields inside the empty cavity resonator are used for the measurement. The Q-value of empty TM010 mode cavity resonators are decided by the intrinsic impedance of the air, the dimension of the cavity, the permeability, and the conductivity of the cavity metal.

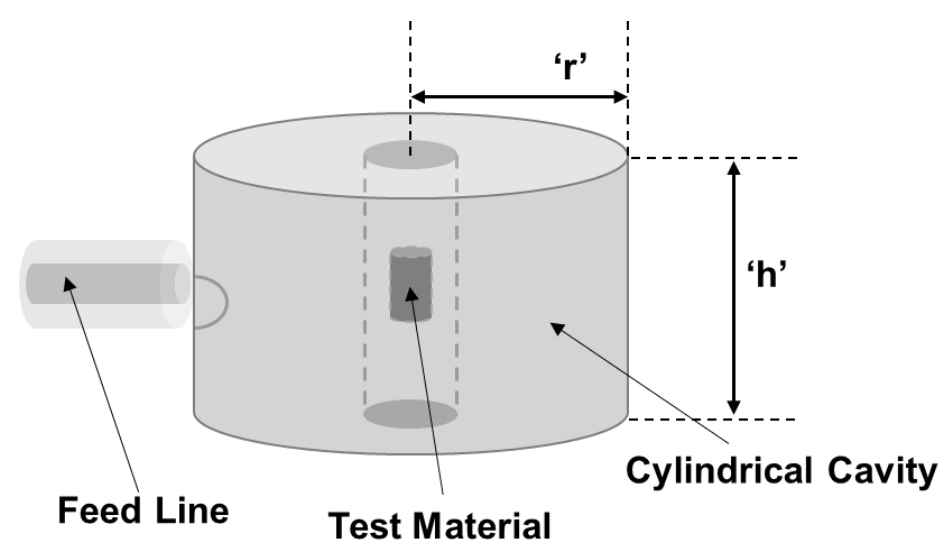

Figure 5 Cavity dimensions $r=150 \mathrm{~mm}, \mathrm{~h}=55 \mathrm{~mm}$

\subsection{Calibration}

For setting up the cavity, an initial calibration has been performed at room temperature. Using the measurement setup, reference measurements for pure Alumina rods Figure 6 (a) of known relative permittivity values $(\mathrm{Er}=9.1)$ has been taken. 
Table 2 Alumina rods sizes for calibration

\begin{tabular}{ccc}
\hline & Sample \#1 & Sample \#2 \\
\hline Length (mm) & $\mathbf{1 7 . 8}$ & $\mathbf{1 7 . 8}$ \\
\hline Diameter (mm) & $\mathbf{4}$ & $\mathbf{6}$ \\
\hline
\end{tabular}

The measured values were compared against the known value to determine the accuracy of the measurement. The resonance of the cavity for the TM010 mode was found at $790 \mathrm{MHz}$, as shown in Figure 2 (b)

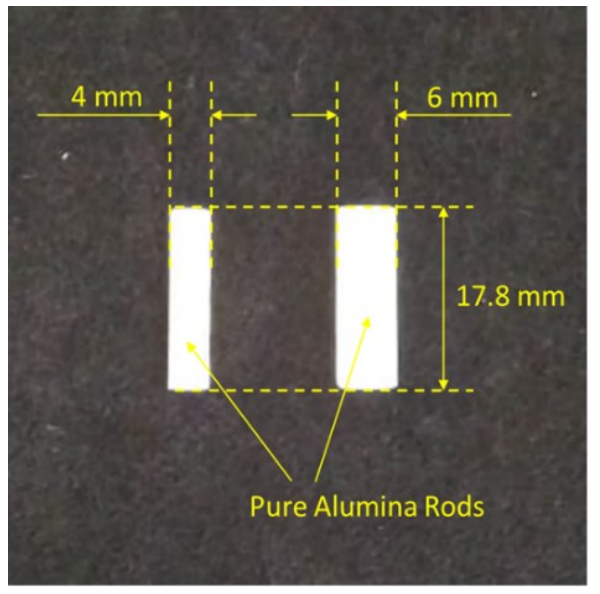

a)

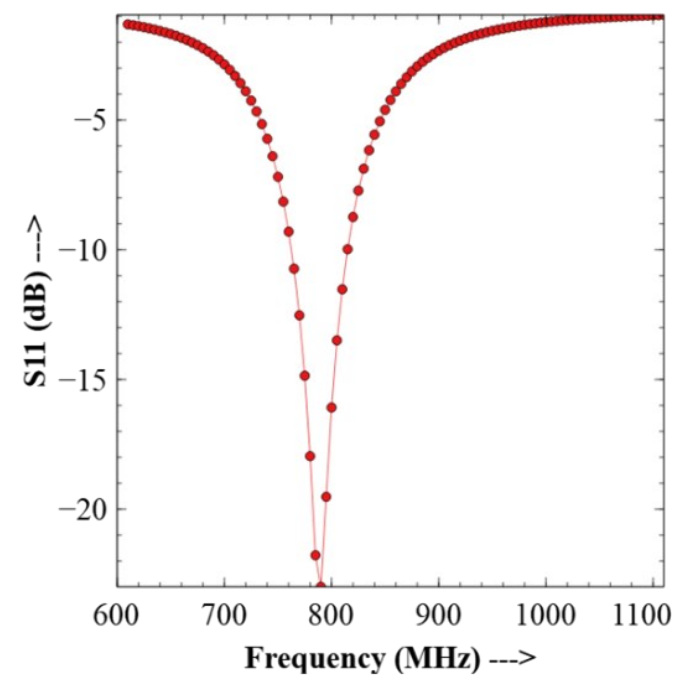

b)

Figure 6 Measured resonant frequency of the fabricated cavity resonator using the VNA

Vector network analyzer measures the change in frequency $(\Delta \mathrm{f})$ and quality factor shift $(\Delta \mathrm{Q})$ between empty cavity and cavity with a sample. The data is fed in the equation (1) \& (2) for computing the dielectric constant ( $\left.\varepsilon^{\prime}\right)$ and loss constant ( $\left.\varepsilon^{\prime \prime}\right)$.

$$
\begin{aligned}
& \varepsilon^{\prime}-1=\frac{f_{c}-f_{s}}{2 f_{s}} \frac{V_{c}}{V_{s}} \ldots \ldots \ldots \ldots . . . e q(2) \\
& \varepsilon^{\prime \prime}=\frac{Q_{c}-Q_{s}}{Q_{c} Q_{s}} \frac{V_{c}}{4 V_{s}} \ldots \ldots \ldots \ldots . . . e q(3)
\end{aligned}
$$

Where,

$\mathrm{f}_{\mathrm{c}}=$ Resonant frequency,

$\mathrm{f}_{\mathrm{S}}=$ Resonant frequency with sample,

$\mathrm{Q}_{\mathrm{c}}=$ Quality factor of empty cavity,

$\mathrm{Q}_{\mathrm{S}}=$ Quality factor with sample,

$\mathrm{V}_{\mathrm{c}}=$ Cavity Volume,

$\mathrm{V}_{\mathrm{S}}=$ Sample Volume. 
Multiple room temperature measurements have been taken using pure Alumina rods. The measured values of the dielectric constant for the rods is shown in Figure 7.

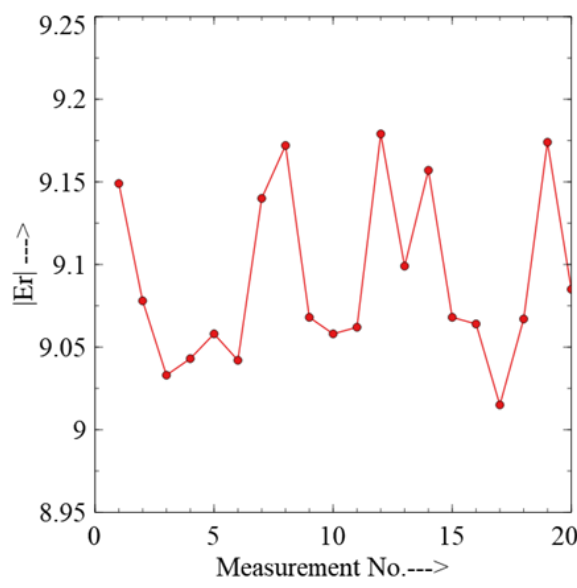

a)

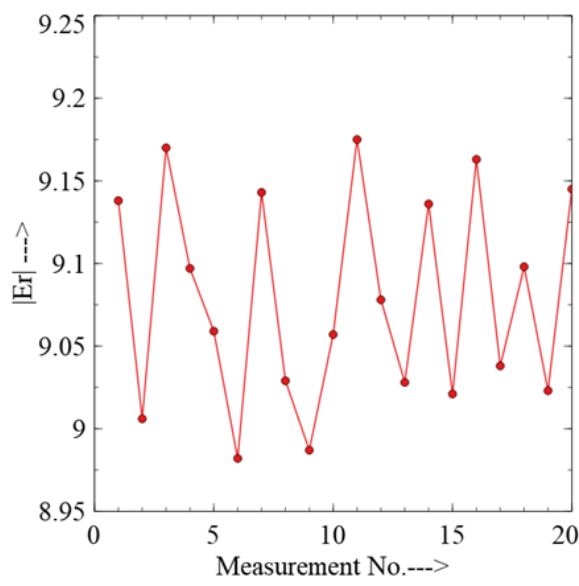

b)

Figure 7 Measured dielectric constant of pure alumina rods $4 \mathrm{~mm}$ (b) $6 \mathrm{~mm}$

Table 3 Measurment summary for the alumina rods

\begin{tabular}{lccc}
\hline Sample Size & Mean & Error & $\begin{array}{c}\text { Std. } \\
\text { Deviation }\end{array}$ \\
\hline $\mathbf{4 m m}$ & 9.103 & $+0.033 \%$ & 0.051 \\
\hline $\mathbf{6 m m}$ & 9.079 & $-0.231 \%$ & 0.064 \\
\hline
\end{tabular}

The measurements provided in Table 3 show high accuracy with an error of $0.033 \%$ and $0.231 \%$ for the rod diameter of $4 \mathrm{~mm}$ and $6 \mathrm{~mm}$ respectively. Hence it can be inferred that the developed experimental setup can measure dielectric samples with very high accuracy.

\subsection{Measurement for coal sample}

An amorphous silica sample holder tube ( 4 mm inner diameter and $\sim 60 \mathrm{~cm}$ long) was used in this case to hold the crushed pelletized coal sample inside the cavity. A thin quartz base is inserted by a glassblower partway up the sample-holder tube to make the top of the tube into the equivalent of a long test-tube. A small hole was put in the base $(\sim 1.3$ mm diameter) to allow the cover gas (UHP argon) to flow up past the sample during the run.

Sample preparation and calibration techniques are often tailored to the properties of the specific samples. In the present case, the coarse grains of coal were ground with a Mortar 
$\&$ Pestle to make a finer powder, which was then pressed into pellets in a uniaxial press at 21,000 psi. Surgical gloves or tweezers were used when working with the clean samples to assure continued cleanliness.

The sample mass was determined before the measurement, and, along with the sample dimensions, is used to calculate an initial bulk density.

\section{The initial sample parameters were:}

a) diameter $-3.64 \pm 0.05 \mathrm{~mm}$

b) length $-11.5 \pm 0.20 \mathrm{~mm}$

c) mass $-0.151 \pm 0.003 \mathrm{~g}$

d) color/appearance - two stacked black rods

e) room temperature initial density $-1.26 \pm 0.06 \mathrm{~g} / \mathrm{cc}$

The steps involved in the measurement were:

Step 1: Collecting and weighing coal: Unpacking the received samples and weighing 2-5 $\mathrm{g}$ coal (or as required) for pellet formation.

Step 2: Preparing mold for pellet formation: Crushing (if needed) with mortar and pestle arrangement and filling the mold cavity with coal.

Step 3: Pressing of coal powder in a mold with a manual hydraulic press to form a cylindrical pellet as in Figure 8.

Step 4: Removing pellet from mold and transfering it to the measurement setup

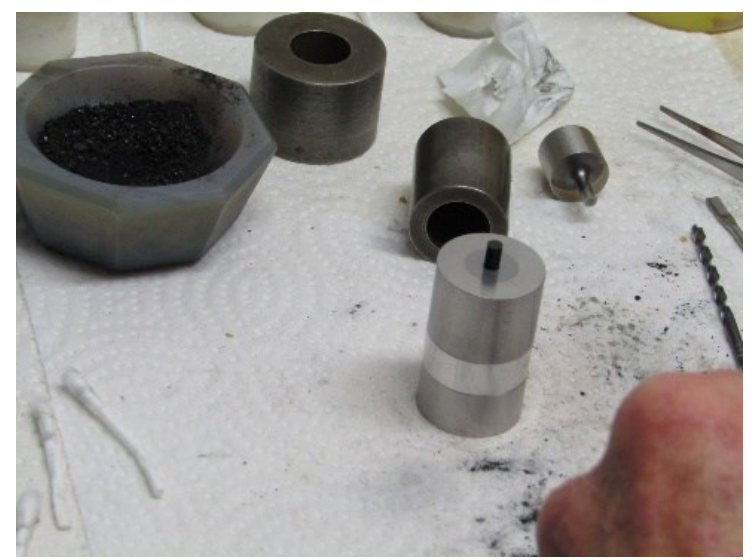

Figure 8 Pellet formation by pressing of coal powder in a mold

\subsection{Measurement procedure}

The procedure starts with calibration with a copper sample and then with an empty sample holder. The piece is then inserted into the furnace for heating. The heated sample is transferred to the cavity (Figure 9) for a specific amount of time and temperature, and VNA 
data collection is done. The above processes happen in an Argon atmosphere. The sample is then extracted, and data is collected on a USB drive. Data for some of these steps are mentioned in next section, where an example of a coal sample is given.

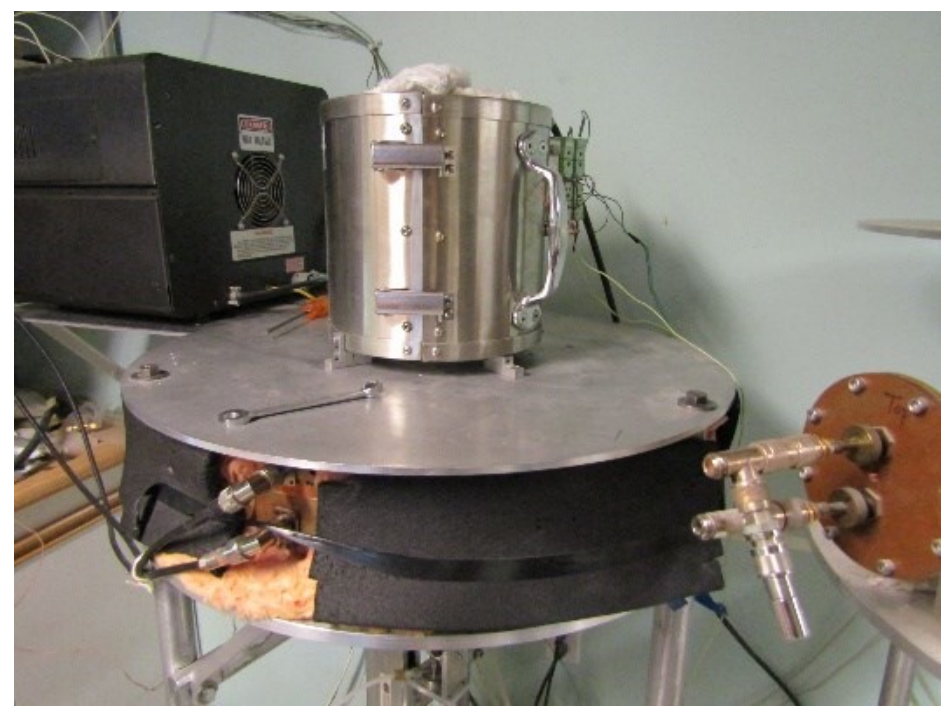

Figure 9 Insulated copper cavity with split furnace mounted on it

\section{Results}

The absolute value of electrical permittivity has been plotted against the multiple measurement iterations in figure 10. The mean value $|\mathrm{Er}|$ for the 100 measurements was 4.195, while the standard deviation obtained was 0.059 at room temperature $\left(25^{\circ} \mathrm{C}\right)$. This sample has been taken from the first source.

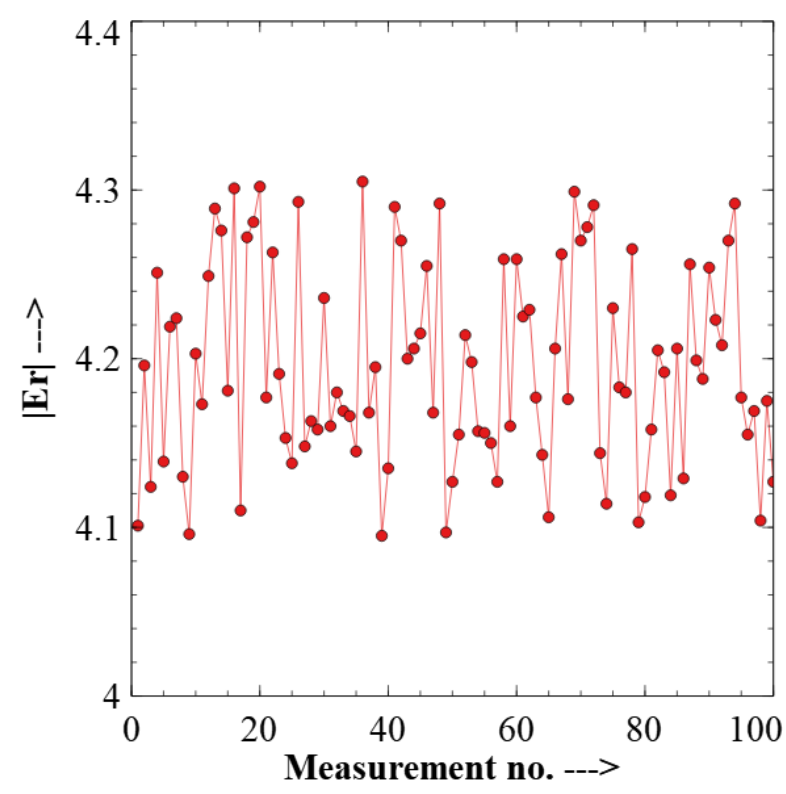

Figure 10 Room temperature $\left(25^{\circ} \mathrm{C}\right)$ Dielectric constant of the coal sample. 
After obtaining the dielectric constant values at $\left(25^{\circ} \mathrm{C}\right)$ the heating cycle started for the coal sample from source1 using a box furnace. The measurement procedure consisted of increasing the sample temperature in steps of $\sim 50^{\circ} \mathrm{C}$ up to $600^{\circ} \mathrm{C}$, and then reducing the temperature in $50^{\circ} \mathrm{C}$ steps to the room temperature. The results obtained from these measurements are as shown in Figure 11. After each temperature step, the temperature is held for $\sim 5$ minutes to allow the temperature in the sample to stabilize and become uniform. After finishing each run, the mass was again determined (when possible) to within \pm 0.002 gm. The sample was then removed from the holder, the holder re-inserted into the system, and the empty holder values measured in $50^{\circ} \mathrm{C}$ steps up to high temperatures to test for contamination and background subtraction. A run has been done in $50^{\circ} \mathrm{C}$ steps up to $600^{\circ} \mathrm{C}$ with an empty sample holder to determine the shape of the "empty holder" subtraction values.

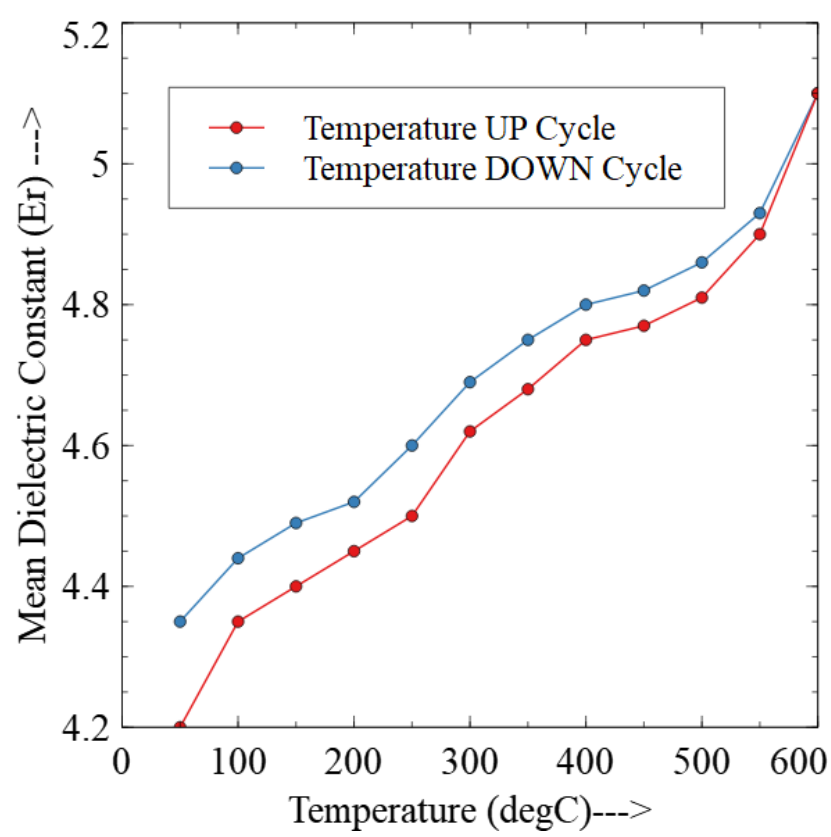

Figure 11 Dielectric constant of coal sample with respect to the temperature.

The coals charged in the blast furnace may come from different sources/mines and possess different properties. These properties include dielectric properties of coals along with their other physical and chemical properties. In continuation, coal samples from the various location were tested in the described setup, and the corresponding values were plotted in Figure 12. 


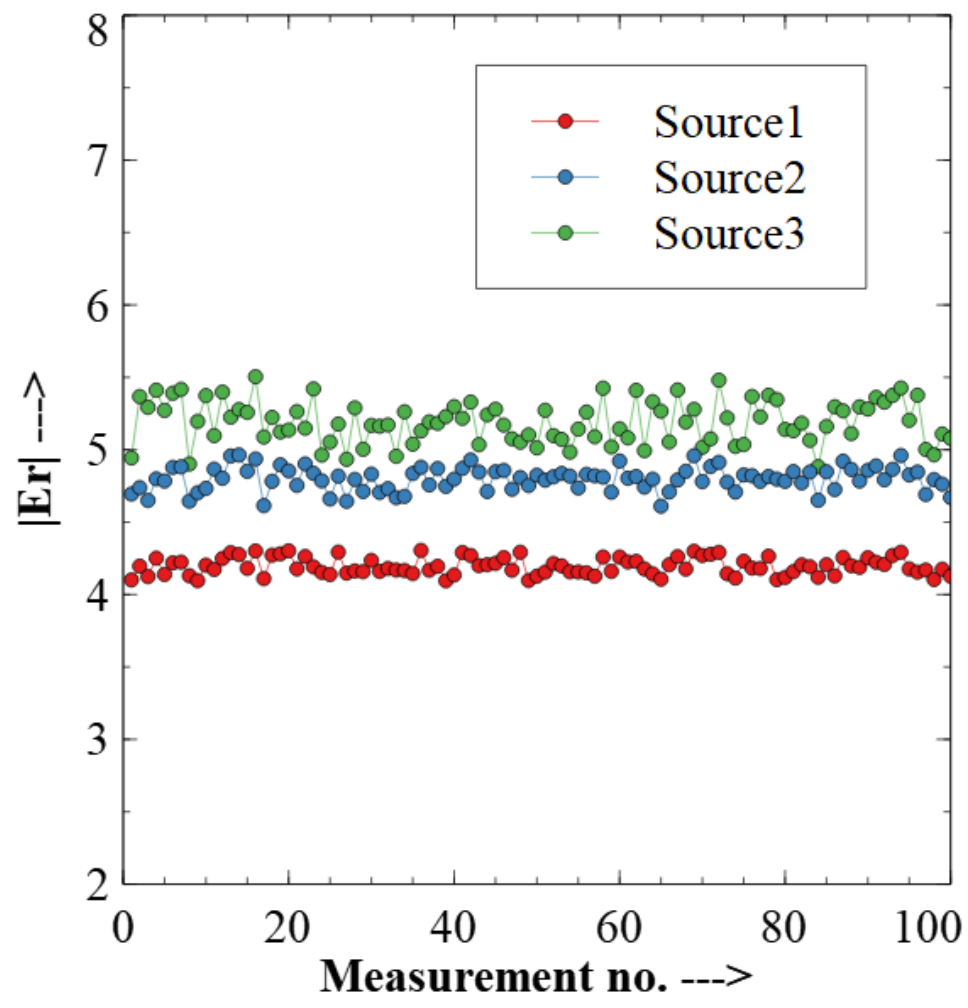

Figure 12 Comparison of the dielectric constant of coal samples from various sources

Table 4. Measurment summary for the the coal samples from different sources

\begin{tabular}{lcc}
\hline Sample Size & Mean & $\begin{array}{c}\text { Std. } \\
\text { Deviation }\end{array}$ \\
\hline Source 1 & $\mathbf{4 . 1 9 5}$ & $\mathbf{0 . 0 5 9}$ \\
\hline Source 2 & $\mathbf{4 . 7 9 8}$ & $\mathbf{0 . 0 8 0}$ \\
\hline Source 3 & $\mathbf{5 . 1 9 2}$ & $\mathbf{0 . 1 4 5}$ \\
\hline
\end{tabular}

\section{Conclusion}

The microwave cavity resonator-based experimental setup designed measured the dielectric constant with very high accuracy. The setup has been used to measure the dielectric properties of coal for temperature up to $600^{\circ} \mathrm{C}$. It has been observed that the dielectric contact increases with the increase in temperature. Further coal samples from different sources were considered for measurement. The results show that the coal samples from different sources showcase different dielectric behavior. The information provided in this paper can assist in designing, modeling of the blast furnace for microwave application like RADAR measurements which is essential to determine the burden profile distribution. 


\section{Acknowledgment}

This paper and the research behind it would not have been possible without the exceptional support of the members at ICT Lab, Automation Division, TATA Steel, India. My colleagues and my superiors have looked over my transcriptions and answered with unfailing patience numerous questions.. I am also grateful for the insightful comments and. the generosity and expertise of one and all that have improved this study in innumerable ways and saved me from many errors.

\section{Bibliography}

[1] M Greedes, H Toxopeus, and C Van Der Vliet, “Modern Blast Furnace Ironmaking[M]”, 2nd ed.,Ios Press, 2009, pp. 1-6.

[2] L. Hartshorn and W. H. Ward, "The measurement of permittivity and power factor of dielectrics at frequencies from 104 to 108 cps," J. IEEE(London), vol. 79, pp. 567-609, November 1936.

[3] M. G. Broadhurst and A. J. Bur, "Two-terminal dielectric measurements up to $6 \times 108$ Hz,’J. Res. NBS, vol.69C, pp. 165-172, July 1965.

[4] K. J. Bois, L. F. Handjojo, A. D. Benally, K. Mubarak and R. Zoughi, "Dielectric plugloaded two-port transmission line measurement technique for property characterization of granular and liquid materials,” IEEE Trans. Instrum. Meas., vol. 48, no. 6, Dec. 1999.

[5] K. Sudheendran, K C James Raju, M. Ghanashyam Krishna and Anil. K Bhatnagar, "Determination of the complex permittivity of materials using a partially filled wave guide," presented from University of Hyderabad, India.

[6] R. J. Cook, "Microwave cavity methods" in High Frequency Dielectric Measurement (Conf. Proc., March 1972), J. Chamberlain and G. W. Chantry, Eds. Guildford, U.K.: IPC Science and Technology Press, 1973, pp. 12-27.

[7] T. M. Hirvonen, P. Vainikainen, A. Lozowski and A. V. Räisänen, "Measurement of dielectrics at $100 \mathrm{GHz}$ with an open resonator connected to a network analyzer," IEEE Trans. Instrum. Meas., vol. 45, no. 4, August 1996.

[8] R. N. Clarke and C. B. Rosenberg, "Fabry-perot and open resonators at microwave and millimeter wave frequencies, 2 - 300 GHz.” J. Phys. E: Sci. Instrum., vol. 15, pp. 9-24, Jan. 1982. 
[9] R. J. Cook and C. B. Rosenberg, "Measurement of the complex refractive index of isotropic and anisotropic materials at $35 \mathrm{GHz}$ using a free space microwave bridge," J. Phys. D: Appl. Phys., vol. 12, pp. 1643-1652, 1979.

[10] Sample shape correction factors for cavity perturbation measurements. F. Adams, M. De Jong, R. Hutcheon. Journal of microwave power and electromagnetic energy, Vol. 27, No. 3, 1992, p. $131-135$.

[11] The cavity perturbation method for the measurement of the relative dielectric permittivity in the microwave range. S. B. Balmus, G. N. Pascariu, F. Creanga, I. Dumitru, D. D. Sandu. Journal of optoelectronics and advanced materials, Vol. 8, No. 3, June 2006, p. $971-977$.

[12] A system for rapid measurement of RF and microwave properties up to $1400^{\circ} \mathrm{C}$. R. Hutcheon, M. De Jong, F. Adams. Journal of microwave power and electromagnetic energy, Vol. 27, No. 2, 1992, p. $87-92$.

[13] Sample shape correction factors for cavity perturbation measurements. F. Adams, M. De Jong, R. Hutcheon. Journal of microwave power and electromagnetic energy, Vol. 27, No. 3, 1992, p. 131 - 135. 